\title{
Low-Variance Multitaper MFCC Features: a Case Study in Robust Speaker Verification
}

\author{
Tomi Kinnunen, Rahim Saeidi, Member, IEEE, Filip Sedlák, Kong Aik Lee, Johan Sandberg, \\ Maria Hansson-Sandsten, Member, IEEE, and Haizhou Li, Senior Member, IEEE
}

\begin{abstract}
In speech and audio applications, short-term signal spectrum is often represented using mel-frequency cepstral coefficients (MFCCs) computed from a windowed discrete Fourier transform (DFT). Windowing reduces spectral leakage but variance of the spectrum estimate remains high. An elegant extension to windowed DFT is the so-called multitaper method which uses multiple time-domain windows (tapers) with frequencydomain averaging. Multitapers have received little attention in speech processing even though they produce low-variance features. In this paper, we propose the multitaper method for MFCC extraction with a practical focus. We provide, firstly, detailed statistical analysis of MFCC bias and variance using autoregressive process simulations on the TIMIT corpus. For speaker verification experiments on the NIST 2002 and 2008 SRE corpora, we consider three Gaussian mixture model based classifiers with universal background model (GMM-UBM), support vector machine (GMM-SVM) and joint factor analysis (GMM-JFA). Multitapers improve MinDCF over the baseline windowed DFT by relative $20.4 \%$ (GMM-SVM) and $13.7 \%$ (GMM-JFA) on the interview-interview condition in NIST 2008. The GMM-JFA system further reduces MinDCF by $18.7 \%$ on the telephone data. With these improvements and generally noncritical parameter selection, multitaper MFCCs are a viable candidate for replacing the conventional MFCCs.
\end{abstract}

Index Terms-Mel-frequency cepstral coefficient (MFCC), multitaper, speaker verification, small-variance estimation

\section{INTRODUCTION}

$\mathbf{F}$ EATURE EXTRACTION is the key function of a speech processing front-end. Spectral features computed from the windowed discrete Fourier transform (DFT) [1] or linear prediction (LP) models [2] are used in most of the frontends. The DFT and LP models perform reasonably well under clean conditions but recognition accuracy degrades severely under changes in environment and channel since the shortterm spectrum is subjected to many harmful variations. In

Copyright (C2012 IEEE. Personal use of this material is permitted.

T. Kinnunen and F. Sedlák and R. Saeidi are with the School of Computing, University of Eastern Finland, Joensuu, Finland (email: \{tkinnu,fsedlak,rahim\}@cs.joensuu.fi). R. Saeidi is with the Radboud University Nijmegen, Netherlands (e-mail: rahim.saeidi@let.ru.nl). J. Sandberg and M. Hansson-Sandsten are with Mathematical Statistics, Centre for Mathematical Sciences, Lund University, Lund, Sweden (email: \{sandberg, sandsten\}@maths.Ith.se). K.A. Lee and H. Li are with the Human Language Technology of the Institute for Infocomm Research (I2R), Singapore (email: \{kalee, hli\}@i2r.a-star.edu.sg).

The work of T. Kinnunen was supported by the Academy of Finland (project no. 132129) and the works of R. Saeidi and H. Li were supported by Nokia foundation. Computing services from CSC - IT Center for Science were used for the speaker verification experiments (project no uef4836). The first author would like to thank professor Juha Alho at UEF for useful suggestions to Section IV this paper, we focus on one of the most successful techniques, the mel-frequency cepstral coefficients (MFCCs), that were introduced three decades ago [3] and are extensively used in speaker and language recognition, automatic speech recognition, emotion classification, audio indexing and, with certain modifications, even in speech synthesis and conversion applications. There is no doubt that the way we derive MFCC features has great impact on the performance of many speech processing applications.

There have been many attempts to enhance the robustness of MFCC features. Several techniques have demonstrated effective ways to normalize the MFCC features by using the statistics of the MFCC temporal trajectory. For example, cepstral mean and variance normalization (CMVN) [4], RASTA filtering [5], temporal structure normalization [6], feature warping [7], and MVA processing [8] are commonly used for enhancing MFCC robustness against additive noises and channel distortions. The specific configuration and order of chaining them depends, however, on the target application. Such techniques obtain the statistics either from the run-time signals themselves or from some training data. Therefore, they require either delayed processing or off-line modeling. In this paper, we would like to study a new way to derive MFCC features, with which we reduce the MFCC estimation variance without relying on any statistics beyond a speech frame.

From a statistical point of view, the common MFCC implementation based on windowed DFT is suboptimal due to high variance of the spectrum estimate [10]. To elaborate on this, imagine that, for every short-term speech frame there exists an underlying random process which generates that particular frame; an example would be an autoregressive (AR) process driven with random inputs but with fixed coefficients. For speech signals, we imagine that there exists a speaker- and phoneme-dependent vocal tract configuration from which the actual speech sounds are generated from. A spectrum estimator with high variance then implies that, for the same underlying random process (e.g., two non-overlapping parts of the very same vowel sound), the estimated spectra and MFCCs may vary considerably.

In speaker verification [11], uncertainty in features is modeled by the variances in the Gaussian mixture models (GMMs) [12] and, recently, by subspace models of speaker and session variabilities in a supervector space [13]-[19]. However, if the MFCCs themselves are estimated with smaller variance, one should expect the subsequent speaker and session variability models to exhibit less random variation as well. Using lowvariance spectrum estimators has already been demonstrated to 


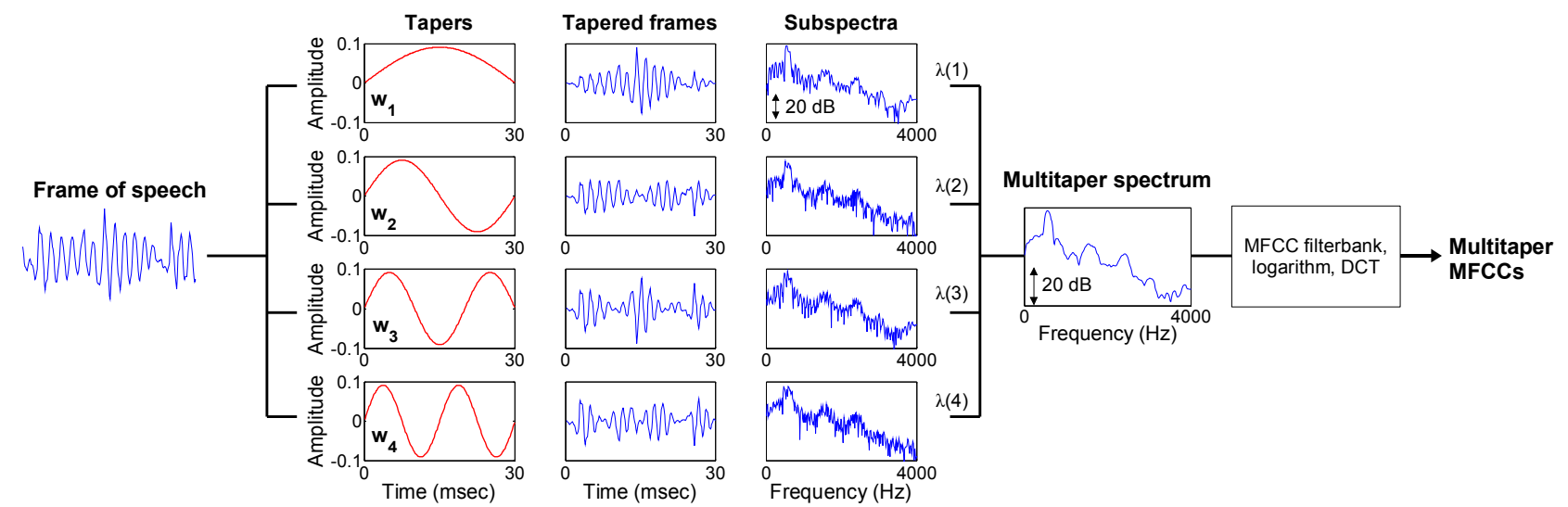

Fig. 1. Multitaper method uses multiple windows (tapers) leading to different subspectra, whose weighted average forms the spectrum estimate and leads to robust MFCCs. For visualization, spectra are shown in dB scale but computations are based on linear values. The tapers are from the SWCE method [9].

enhance performances of voice activity detection (VAD) [20], [21], speech enhancement [22] and speech recognition [23], to give a few examples.

The particular small-variance method adopted in this paper is based on multitapers, as illustrated in Fig. 1. The multitaper method [24]-[27], as a simple and elegant extension of the conventional windowed DFT, uses multiple window functions (aka tapers) with weighted frequency-domain averaging to form the spectrum estimate [24], [25], [27]. The tapers are designed to give approximately uncorrelated spectrum estimates so that averaging them reduces the variance. More specifically, the side-lobe leakage effect in the conventional windowed DFT is partially suppressed by multitapering [28], [29]. The wellknown Welch's method [30] is a special case of the multitaper technique with identically shaped but time-shifted tapers. Thus, in Welch's method, the subspectra are uncorrelated because they are computed from different segments. The multitapers applied in this paper, in contrast, are fully overlapping in time but their shapes are designed so that they have only small overlap in frequency domain [10], [24]. Conceptually, multitapering also shares some similarity with smoothing the DFT estimate using frequency-domain convolution (e.g. [10]), but generally these are not mathematically equivalent.

The multitaper method of spectrum estimation was introduced around the same time as the MFCCs [24] but has found little use in speech processing so far [22], [31], [32]. This might be due to previously unstudied statistical properties of multitaper MFCCs and availability of different multitaper variants to choose from [9], [24]-[26]. Additionally, due to mostly theoretically focused treatments of the topic [10], [24], [25], practitioners may have had difficulties in implementing and choosing the control parameters in a typical recognition application.

Since the statistical properties of the multitaper MFCCs - briefly summarized in Section III - are recently analyzed [27] and further, we got encouraging preliminary speaker verification results in [33], we were curious to explore the technique further. In Section IV we carry out detailed evaluation of multitaper bias and variance using simulated random processes on the TIMIT corpus. Importantly, in Sections V and
VI we extend and complement the preliminary GMM-UBM results of [33] using two high-performance classifiers, GMM supervector with support vector machine (GMM-SVM) [13], [34] and GMM with joint factor analysis technique (GMMJFA) including integrated speaker and intersession variability modeling [15], [35], [36]. To sum up, the main purpose of this paper is to review, collect and extend our recent work on the use of multitapers in speech processing with application to speaker verification. We provide sample implementation and recommendations for setting the control parameters.

\section{Computing the Multitaper MFCCs}

Let $\mathbf{x}=[x(0) \ldots x(N-1)]^{\mathrm{T}}$ denote one frame of speech of $N$ samples. The most popular spectrum estimate in speech processing, windowed discrete Fourier transform (DFT), is given by

$$
\hat{S}(f)=\left|\sum_{t=0}^{N-1} w(t) x(t) \mathrm{e}^{-i 2 \pi t f / N}\right|^{2},
$$

where $i=\sqrt{-1}$ is the imaginary unit and $f=$ $0,1, \ldots, N-1$ denotes the discrete frequency index. Here $\mathbf{w}=\left[\begin{array}{lll}w(0) & \ldots w(N-1)\end{array}\right]^{\mathrm{T}}$ is a time-domain window function which usually is symmetric and decreases towards the frame boundaries. In this study, we choose the most popular window in speech processing, the Hamming window, with $w(t)=0.54-0.46 \cos (2 \pi t / N)$.

From a statistical perspective, the use of a Hamming-type of window reduces the bias of the spectrum estimate, i.e. how much the estimated value $\hat{S}(f)$ differs from the true value $S(f)$, on average. But the estimated spectrum still has high variance. To reduce the variance, multitaper spectrum estimator [10], [24], [26] can be used:

$$
\hat{S}(f)=\sum_{j=1}^{K} \lambda(j)\left|\sum_{t=0}^{N-1} w_{j}(t) x(t) \mathrm{e}^{-i 2 \pi t f / N}\right|^{2} .
$$

Here, $K$ multitapers $\mathbf{w}_{j}=\left[w_{j}(0) \ldots w_{j}(N-1)\right]^{\mathrm{T}}$, where $j=1, \ldots, K$, are used with corresponding weights $\lambda(j)$. The multitaper estimate is therefore obtained as a weighted 


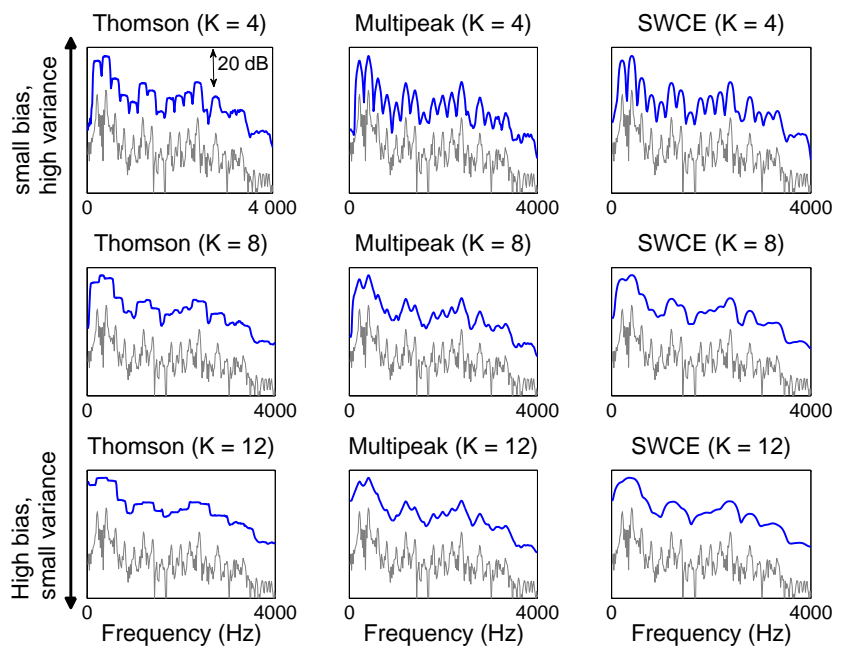

Fig. 2. Typical multitaper spectra for the methods used in this paper. The lower thin lines (gray) show the Hamming-windowed DFT spectrum as a reference. The spectra have been shifted by $20 \mathrm{~dB}$ for visualization.

average of $K$ subspectra (Fig. 1). The windowed DFT (1) is obtained as a special case when $K=1$ and $\lambda=1$.

\section{A. Choice of the Tapers}

A number of different tapers have been proposed for spectrum estimation, such as Thomson [24], sine [25] and multipeak tapers [26]. For cepstrum analysis, the sine tapers are applied with optimal weighting in [9]. Each type of taper is designed for some given type of (assumed) random process; as an example, Thomson tapers are designed for flat spectra (white noise) and multipeak tapers for peaked spectra (such as voiced speech). In practice, many multitapers work well even though designed for another process. For instance, the Thomson tapers [24], designed for white noise, tend to perform well for any smooth spectrum.

In general, the tapers are designed so that the estimation errors in the subspectra will be approximately uncorrelated, which is the key to variance reduction. It is out of the scope of this paper to describe the details of finding optimal tapers. For theoretical treatment, we point the reader to [10], [24] while [9], [25], [26], [37] provide more concise discussions. At the Appendix of this paper, we point to practical MATLAB implementations. In short, the solution is obtained from an eigenvalue problem where the eigenvectors and -values correspond to the tapers and their weights, respectively. The tapers considered in this paper are all computed off-line without any data-adaptive training process and applied to all speech utterances.

Fig. 2 shows, for a single voiced speech frame, examples of the three multitaper methods considered in this study, Thomson [24], multipeak [26] and sine-weighted cepstrum estimator (SWCE) [9]. Each panel shows the multitaper spectrum (upper thick line) along with Hamming-windowed DFT estimate (lower thin line). All the three multitaper methods produce smoother spectrum compared to the Hamming method, because of variance reduction. Thomson produces a staircase-like spectrum, multipeak a spectrum with sharper peaks and SWCE a compromise between these two methods. In this example, for a small number of tapers, say $K \leq 4$, all the three methods preserve both the harmonics (due to the voice source) and the spectral envelope (due to the vocal tract). For a high number of tapers, say $K \geq 8$, the harmonics gets smeared out. The optimum number of tapers is expected to depend on the target application. In speaker recognition, both the voice source and vocal tract filter are found to be useful, thus we expect to get the best results using a relatively small number of tapers.

\section{B. Computational Complexity and Periodogram Smoothing}

The windowed periodogram in (1) can be computed using fast Fourier transform (FFT) of complexity $\mathcal{O}(N \log N)$. Since the multitaper estimator (2) requires $K$ FFTs, the complexity of the direct implementation is $\mathcal{O}(K N \log N)$, which might become a critical design consideration under low-resource platforms. Luckily, when the tapers are sinusoids as in [25] and the SWCE method [9], complexity can be reduced. Indeed, the the $j$ th sine taper can be written using Euler's formula as,

$$
w_{j}(t)=\sin \left(2 \pi f_{j} t\right)=\frac{1}{2 i}\left\{e^{i 2 \pi f_{j} t}-e^{-i 2 \pi f_{j} t}\right\} .
$$

Thus, DFT of the windowed data segment $x(t) w_{j}(t)$ is,

$$
\mathcal{F}\left\{x(t) w_{j}(t)\right\}=\frac{1}{2 i}\left\{X\left(f-f_{j}\right)-X\left(f+f_{j}\right)\right\},
$$

where $\mathcal{F}\{\cdot\}$ denotes the DFT operator and $X(f)=\mathcal{F}\{x(n)\}$. Substituting this to the multitaper spectrum estimator (2) and simplifying leads to,

$$
\begin{aligned}
\hat{S}(f) & =\frac{1}{4} \sum_{j=1}^{K} \lambda(j)\left\{\left|X\left(f-f_{j}\right)\right|^{2}+\left|X\left(f+f_{j}\right)\right|^{2}\right. \\
& \left.-2 X\left(f-f_{j}\right) X^{*}\left(f+f_{j}\right)\right\} .
\end{aligned}
$$

This consists of computing $X(f)$ by one FFT, followed by the three frequency-domain smoothing terms of complexity $\mathcal{O}(K N)$, thus totaling $\mathcal{O}(N \log N+K N)$ steps. Since typically $K \ll N$, this is usually faster than the direct implementation (2).

A popular method for producing smooth spectrum estimates, periodogram smoothing, is to convolve the unwindowed (raw) periodogram with a suitable frequency-domain smoothing kernel, which has also complexity $\mathcal{O}(N \log N+K N)$. Note that the first sum term in (5) is, in fact, the convolution of $|X(f)|^{2}$ with kernel $\left\{\lambda_{1}, \lambda_{2}, \ldots, \lambda_{K}\right\}$. However, because of the two additional terms, the methods are not equivalent. In our speaker verification experiments, we will also provide experiments with periodogram smoothing.

\section{Bias AND VARiAnce OF Multitaper Estimators}

To understand the bias and variance trade-off better, we consider the variance and spectral resolution of the single- and multi-taper methods. For the windowed DFT (1), the variance is usually approximated as [10],

$$
V[\hat{S}(f)] \approx S^{2}(f) .
$$


The spectral resolution, that is, the frequency spacing under which two frequency components cannot be separated, is approximately $B_{w}=1 / N$ for the rectangle window but $B_{w}=2 / N$ for the Hamming window. Note also that (6) does not depend on the frame length $N$ and thus, including more samples in a frame will not reduce the variance.

For the multitaper spectrum estimator (2), the spectral resolution is approximately $B_{w}=(K+2) / N$ which is the spectral resolution parameter used in the design of the Thomson [24] and multipeak [26] tapers. The variance can be approximated as,

$$
V[\hat{S}(f)] \approx \frac{1}{K} S^{2}(f) .
$$

This result is analogous to the well-known result that variance of the mean of sample of size $K$ is inversely proportional to $K$ [4, p. 82]. The formula (7) is approximately valid also for the Welch's method [30] with $50 \%$ overlap between the windows [10].
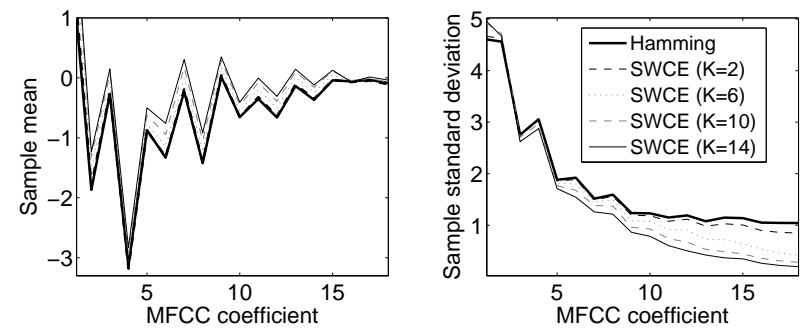

Fig. 3. Multitapers help in reducing variance of the higher-order MFCCs, but without modifying the mean value much.

Note that up to this point we have only considered variance and bias in spectral and not MFCC domain. Intuitively it is easy to understand, that if the spectrum is estimated with low bias and low variance, the resulting MFCC vector will also have low bias and variance. Using vector notation, the MFCC vector $\mathbf{c}$ is related to the (true) spectrum vector $\mathbf{s}=$ $[S(0) \ldots S(N-1)]^{\mathrm{T}}$ by $\mathbf{c}=\frac{1}{M} \boldsymbol{\Phi}^{\mathrm{H}} \log (\mathbf{M s})$, where $M$ is the number of filters in the filter-bank $\mathbf{M} \in \mathbb{R}^{M \times N}$, the logarithm operates element-wise and $\boldsymbol{\Phi}$ is the $M$-by- $M$ Fourier matrix with the $(a, b)^{\text {th }}$ element: $\mathbf{\Phi} \triangleq\left\{\mathrm{e}^{-i 2 \pi(a-1)(b-1) / M}\right\}_{a b}$. Bias of cepstral coefficients has been studied in [38], whereas

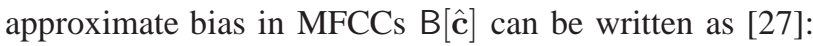

$$
\mathrm{B}[\hat{\mathbf{c}}] \approx \frac{1}{M} \boldsymbol{\Phi}^{\mathrm{H}}\left(\log \left(\frac{\mathbf{M E}[\hat{\mathbf{s}}]}{\mathbf{M} \mathbf{s}}\right)-\frac{\operatorname{diag}\left(\mathbf{M V}[\hat{\mathbf{s}}] \mathbf{M}^{\mathrm{T}}\right)}{2(\mathbf{M E}[\hat{\mathbf{s}}])^{2}}\right)
$$

Here, the division operates element-wise, $\hat{\mathbf{s}}=$ $[\hat{S}(0) \ldots \hat{S}(N-1)]^{\mathrm{T}}$ denotes the estimated spectrum using multitapers (2), $E[\hat{\mathbf{s}}]$ denotes the expected value of $\hat{\mathbf{s}}$, $\mathrm{V}[\hat{\mathbf{s}}]$ denotes the covariance matrix of the spectrum estimate and $(\cdot)^{\mathrm{H}}$ stands for conjugate transpose. Both the expected value $\mathrm{E}[\hat{\mathbf{s}}]$ and the covariance matrix $\mathrm{V}[\hat{\mathbf{s}}]$ (see [10], [27] for details) depend on the covariance matrix $\mathbf{R}$ of the random process and hence, on the true spectrum $\mathbf{s}$.

The covariance matrix of the estimated MFCC vector using multitapers can be approximated as [27],

$$
\mathrm{V}[\hat{\mathbf{c}}] \approx \frac{1}{M^{2}} \boldsymbol{\Phi}^{\mathrm{H}} \frac{\mathrm{MV}[\hat{\mathbf{s}}] \mathbf{M}^{\mathrm{T}}}{\operatorname{ME}[\hat{\mathbf{s}}] \mathrm{E}[\hat{\mathbf{s}}]^{\mathrm{T}} \mathbf{M}^{\mathrm{T}}} \boldsymbol{\Phi} .
$$

The bias and variance of the MFCC estimator depend on the true spectrum of the process. As this is usually unknown, it is impossible to use these formulas directly. However, a general rule is that by increasing the number of tapers, we can reduce the variance of the spectrum estimate, hence making the spectrum estimate more robust across random variations. As an example, Fig. 3 shows the sample mean and standard deviations of conventional and multitaper MFCCs for one speech utterance in the NIST 2002 corpus. The mean vectors in this example differ mostly by an additive constant, whereas the variances of the higher order MFCCs (beyond the $5^{\text {th }}$ MFCC) are significantly reduced due to multitapering.

\section{Numerical EVAluation of Bias AND VARiance of MULTITAPER MFCC ESTIMATORS}

\section{A. Monte Carlo Computation of Bias and Variance Using Known Autoregressive Models}

We would like to know how much the estimated MFCCs differ from the true MFCCs. As is common in the evaluation of nonparametric spectrum estimators, we consider a parametric model with known parameters as a ground truth. Due to their success in spectral modeling of speech signals [2], we consider autoregressive $\operatorname{AR}(p)$ random process,

$$
x(t)=-\sum_{m=1}^{p} a_{m} x(t-m)+\varepsilon(t),
$$

where $\left\{a_{m}\right\}_{m=1}^{p}$ are the known AR coefficients and $\varepsilon(t) \sim$ $\mathcal{N}(0,1)$ are i.i.d. samples of the driving white noise sequence. The corresponding $\operatorname{AR}(p)$ spectrum (sampled at discrete data points $f=0,1, \ldots, N-1$ ) is given by,

$$
S_{\mathrm{AR}(p)}(f)=\frac{1}{\left|1+\sum_{m=1}^{p} a_{m} \exp (-i 2 \pi f m / N)\right|^{2}} .
$$

Thus, given the known parameters $\left\{a_{m}\right\}_{m=1}^{p}$, we can simulate a specific realization $\mathrm{x}$ of the random process using (10). Applying windowed DFT, multitaper or any other spectrum estimator on $\mathbf{x}$ produces an estimate $\hat{S}(f)$ of the spectrum (11). Depending on the random input $\varepsilon(t)$ in (10), the estimate will be different each time. We are concerned in how the estimated MFCC vector $\hat{\mathbf{c}}$ (computed from $\hat{S}(f)$ ) differs from the ground-truth MFCC vector $\mathbf{c}^{\mathrm{AR}(\mathrm{p})}$ (computed from (11)) on average. To this end, we consider the three well-known descriptors of any estimator - bias, variance and mean square error (MSE):

$$
\begin{aligned}
\mathrm{B}[\hat{\mathbf{c}}] & =\mathrm{E}[\hat{\mathbf{c}}]-\mathbf{c}^{\mathrm{AR}(\mathrm{p})} \\
\mathrm{V}[\hat{\mathbf{c}}] & =\mathrm{E}\left[\hat{\mathbf{c}}^{2}\right]-(\mathrm{E}[\hat{\mathbf{c}}])^{2} \\
\mathrm{MSE}[\hat{\mathbf{c}}] & =\mathrm{E}\left[\left(\hat{\mathbf{c}}-\mathbf{c}^{\mathrm{AR}(\mathrm{p})}\right)^{2}\right]
\end{aligned}
$$

where we introduced shorthand notation $\mathbf{z}^{2}=\operatorname{diag}\left(\mathbf{z z}^{\mathrm{T}}\right)$ for vector $z$. MSE further links the bias and variance as $\operatorname{MSE}[\hat{\mathbf{c}}]=\mathrm{B}[\hat{\mathbf{c}}]^{2}+\mathrm{V}[\hat{\mathbf{c}}]$. To compute the bias and variance for a single random process (one set of $a_{k} \mathrm{~s}$ ), we approximate 

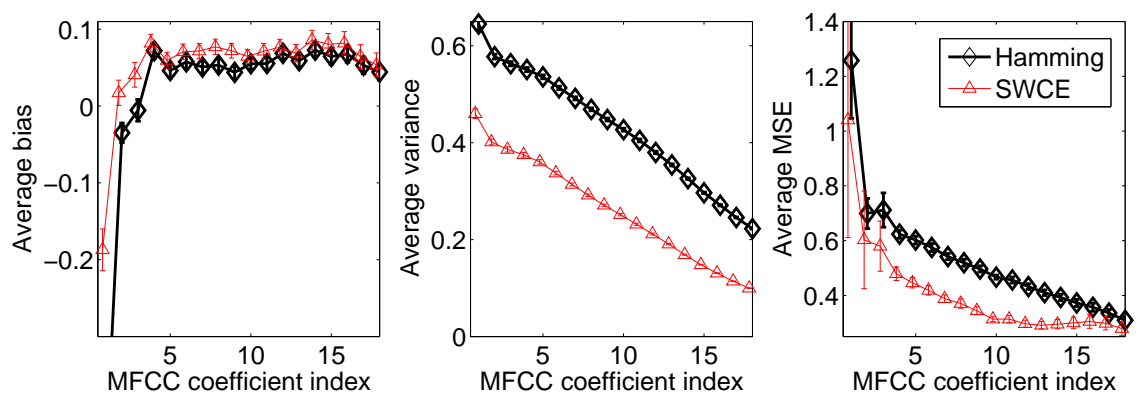

Fig. 4. Average MFCC bias, variance and MSE $\left(\mathrm{MSE}=\right.$ bias $^{2}+$ variance $)$ of Hamming and SWCE $(K=4)$ estimators over 2849 different $\mathrm{AR}$ random processes of varying order. Bias, variance and MSE of each random process are computed using 30000 Monte Carlo draws. The errorbars indicate $95 \%$ confidence interval of the mean. For visual clarity, the results for Thomson and multipeak are excluded. While multitapers slightly increase bias for most coefficients, the variance of each coefficient is significantly reduced. The MSE improvements are most prevalent for coefficients $c_{3}$ through $c_{16}$.
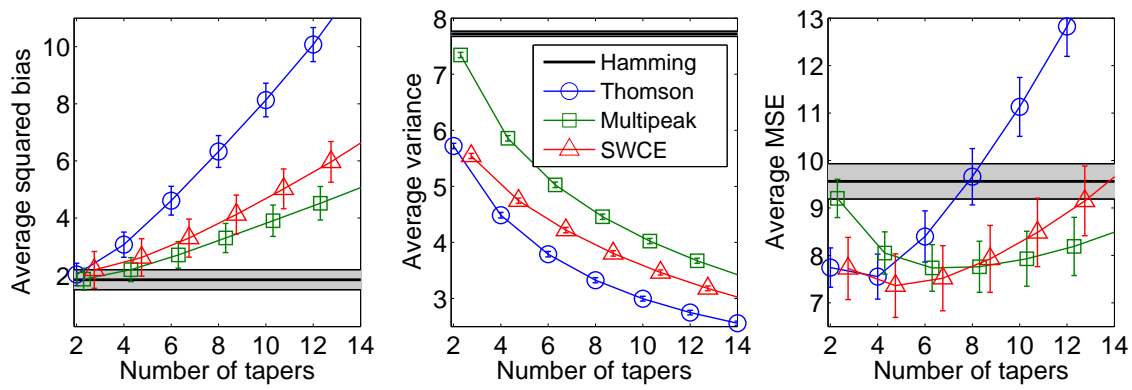

Fig. 5. Similar to Fig. 4 but for squared bias, variance and MSE integrated over all the 18 MFCCs as a function of taper count. The results are shown for $K=2,4, \ldots, 14$ tapers; for visual clarity, multitapers are slightly offset in horizontal direction.

the expectations of random vector $\mathbf{z}$ in (12)-(14) using sample mean as $\mathrm{E}[\mathbf{z}] \approx \frac{1}{N_{\mathrm{MC}}} \sum_{r=1}^{N_{\mathrm{MC}}} \mathbf{z}_{r}$. Here, $N_{\mathrm{MC}}$ is the number of random Monte Carlo draws and $\mathbf{z}_{r}$ corresponds to the vector of the $r$ th random draw. We fix $N_{\mathrm{MC}}=30000$ for which we found the values of (12)-(14) converged so that the Monte Carlo error can be considered neglible.

\section{B. Summarizing Bias, Variance and MSE}

Note that above bias, variance and MSE are defined for a single random process (one set of $a_{k} \mathrm{~s}$ ). Depending on the choice of the coefficients or the order of the AR model $(p)$, one gets different conclusions. As an overall measure, therefore, we are interested on the average bias, variance and MSE over a large number of different random processes (different set of $a_{k} \mathrm{~s}$ and different AR model order $p$ ). This resembles a typical speaker recognition setting where inferences about speaker identity are drawn over a large number of speech frames.

The average MSE vector is given by,

$$
\boldsymbol{\mu}_{\mathrm{MSE}}=\frac{1}{N_{\mathrm{P}}} \sum_{n=1}^{N_{\mathrm{P}}} \operatorname{MSE}\left[\hat{\mathbf{c}}_{n}\right]
$$

where MSE $\left[\hat{\mathbf{c}}_{n}\right]$ indicates MSE (14) of the $n$th random process out from a collection of $N_{\mathrm{P}}$ random processes. We are also interested in whether the difference in the means are statistically significant. To this end, we also compute the confidence interval of the mean for each of the individual coefficients. By denoting the individual dimensions of MSE $\left[\hat{\mathbf{c}}_{n}\right]$ and $\mu_{\mathrm{MSE}}$ by $\operatorname{MSE}_{n}(q)$ and $\mu_{\mathrm{MSE}}(q)$, respectively, we compute the confidence intervals as $\mu_{\mathrm{MSE}}(q) \pm 1.96 \sqrt{\sigma_{\mathrm{MSE}}^{2}(q) / N_{\mathrm{P}}}$ where the MSE variance is given by,

$$
\sigma_{\mathrm{MSE}}^{2}(q)=\frac{1}{N_{\mathrm{P}}-1} \sum_{n=1}^{N_{\mathrm{P}}}\left(\operatorname{MSE}_{n}(q)-\mu_{\mathrm{MSE}}(q)\right)^{2}
$$

for each MFCC feature indexed by $q=1,2, \ldots, 18$. The confidence interval signifies that, with $95 \%$ certainty, the true mean value falls within the confidence bounds. Regarding bias and variance, their means with associated confidence intervals can be similarly computed.

\section{Obtaining the Reference AR Models and MFCCs}

To simulate speech-like AR random processes, we obtain the AR coefficients $a_{k}$ from real speech utterances rather than hand-crafting them. To this end, we pick the common SA1 utterance ("She had your dark suit in greasy wash water all year") from a total number of 77 speakers (59 $0^{x}, 18$ ㅇ) from the training section of the Western dialect (DR7) on the TIMIT corpus. We use the corpus annotations to locate phone boundaries (excluding short phonemes less than $11.25 \mathrm{msec}$ in duration), and compute the AR coefficients of each phone. This set, consisting of $N_{\mathrm{P}}=2849$ phones, is representative of all American English phonemes and phoneme groups. For consistency with the following speaker recognition experiments, we resample the utterances down to $8 \mathrm{kHz}$. To avoid favoring AR models of a particular fixed order, we adapt the AR model order $(p)$ differently for each phoneme. To this end, we use the well-known Schwarz's Bayesian criterion (SBC) [39] implemented in the toolbox of [40]. We set the search limits for the optimum model order as $\left[p_{\min }=1, p_{\max }=40\right]$. 
The MFCCs are extracted using procedure similar to the speaker verification experiments (see subsection V-C). To exclude the effects of application-dependent feature normalizations, we measure the distortions in the lowest 18 base MFCCs, excluding the DC coefficient $c_{0}$. In the recognition experiments (Section VI), however, we utilize a complete front-end with additional RASTA filter, delta features and cepstral mean/variance normalization $(\mathrm{CMVN})$ as a normal practice in speaker verification.

\section{Results}

We first compare the average biases, variances and MSEs of the windowed DFT (Hamming) and SWCE multitaper $(K=4)$ MFCC estimators in Fig. 4; the results for Thomson and multipeak tapers were similar to SWCE and were excluded for visual clarity. Regarding bias of the first cepstral coefficient $c_{1}$, both methods have a large negative bias which is, interestingly, larger in magnitude for Hamming. Regarding the other coefficients, both methods yield positive bias. Hamming introduces generally less bias but SWCE clearly reduces variance of all MFCCs by a wide margin. Regarding MSE of the lowest MFCCs $\left(c_{1}\right.$ through $c_{3}$ ), SWCE yields smaller MSEs but the differences are not significant due to overlapping confidence intervals. However, the intermediate and higherorder MFCCs produce significantly smaller MSEs.

Next we compare bias, variance and MSE integrated over all the 18 MFCC coefficients in Fig. 5 as a function of taper count for all the four estimators. Computations are similar as in Fig. 4 but we replace the vector quantities in (12)-(14) by their corresponding $L_{1}$-norms $\|\cdot\|_{1}$, i.e. the sum of the (absolute value of) individual elements. In the case of bias, we display $L_{1}$-norm of the squared bias. This is natural because $\operatorname{MSE}[\hat{\mathbf{c}}]=\mathrm{B}[\hat{\mathbf{c}}]^{2}+\mathrm{V}[\hat{\mathbf{c}}]$, which helps in better interpreting the relative contributions of bias and variance terms to MSE.

According to Fig. 5, there is a large positive bias for all the four spectrum estimators. This bias is generally larger for the multitaper estimators in comparison to windowed DFT, as expected. But the variance of all three multitaper estimators is significanly smaller than that of Hamming-windowed DFT. For the biases, Hamming $<$ multipeak $<\mathrm{SWCE}<$ Thomson, but the order is reversed for the variances. The compromise measure, MSE, shows nicely convex behaviour; for small number of tapers $K$, the large variance dominates over squared bias, leading to high MSE. For large $K$, similarly, the squared bias dominates and increases MSE. The smallest MSE values are obtained at $K=4$ for Thomson and SWCE and at $K=6$ for multipeak. Behavior of the MSE values suggests that a suitable number of tapers for Thomson might be smaller compared to multipeak and SWCE.

To sum up, the results in Figs. 4 and 5 clearly indicate that multitapers reduce the variance of the MFCC estimates which is useful from generalization point of view. From these application-independent statistical MFCC estimator analyses, suitable $K$ might be on the range $2 \leq K \leq 8$ for typical speech applications utilizing MFCCs, although it will certainly depend on the task at hand; we will now proceed to our target application, speaker verification.
TABLE I

DETAILS OF THE EVALUATION CORPORA AND THE THREE CLASSIFIERS (UBM=UNIV. BACKGROUND MODEL, JFA=JOINT FACTOR ANALYSIS; $\mathrm{NAP}=$ NUIS ANCE ATTRIBUTE PROJECTION; SWB=SWITCHBOARD).

\begin{tabular}{|c|c|c|c|}
\hline & NIST 2002 & \multicolumn{2}{|c|}{ NIST 2008} \\
\hline Speakers & $\overline{139 \sigma^{n}, 191 \%}$ & \multicolumn{2}{|c|}{$10920^{n}, 1649$ q } \\
\hline Gen. trials & 2982 & \multicolumn{2}{|c|}{$15,345^{\mathrm{a}}$} \\
\hline Imp. trials & 39,259 & \multicolumn{2}{|c|}{$56,792^{b}$} \\
\hline Type of data & telephone & \multicolumn{2}{|c|}{ telephone, interview, mic. } \\
\hline Training dur. & $2 \min$ & \multicolumn{2}{|c|}{$3-5 \mathrm{~min}$} \\
\hline Test dur. & $15-45 \mathrm{sec}$ & \multicolumn{2}{|c|}{$3-5 \mathrm{~min}$} \\
\hline & $\begin{array}{c}\text { GMM-UBM } \\
{[12]}\end{array}$ & $\begin{array}{c}\text { GMM-SVM } \\
{[13]}\end{array}$ & $\begin{array}{c}\text { GMM-JFA } \\
{[15],[35],[36]}\end{array}$ \\
\hline $\begin{array}{l}\text { Spec. } \\
\text { subtraction }\end{array}$ & Yes & No & No \\
\hline $\begin{array}{l}\text { Gaussians } \\
\text { per gender }\end{array}$ & 1024 & 512 & 512 \\
\hline $\begin{array}{l}\text { Intersession } \\
\text { compens. }\end{array}$ & - & NAP [34] & $\begin{array}{c}\text { JFA [15], [35], } \\
{[36]}\end{array}$ \\
\hline $\begin{array}{l}\text { Background } \\
\text { data }\end{array}$ & SRE 01 & $\begin{array}{l}\text { SRE 04, 05, } \\
\text { 06, MIXER5 }\end{array}$ & SRE 04 \\
\hline $\begin{array}{l}\text { Eigenchannel } \\
\text { data }\end{array}$ & - & $\begin{array}{l}\text { SRE 04, 06, } \\
\text { MIXER5 }\end{array}$ & $\begin{array}{l}\text { SRE 04, 05, } \\
\text { 06, MIXER5 }\end{array}$ \\
\hline $\begin{array}{l}\text { Eigenvoice } \\
\text { data }\end{array}$ & - & - & $\begin{array}{l}\text { SRE 05, 06, } \\
\text { SWB }\end{array}$ \\
\hline Diag. model & - & - & SRE 04 \\
\hline $\begin{array}{l}\text { Score } \\
\text { normalization }\end{array}$ & $\begin{array}{l}\text { T-norm [41] } \\
\text { SRE } 01\end{array}$ & $\begin{array}{c}\text { ZT-norm [42] } \\
\text { SRE 05, } 06\end{array}$ & $\begin{array}{c}\text { TZ-norm [42] } \\
\text { SRE 05, } 06\end{array}$ \\
\hline
\end{tabular}

a $11540 \operatorname{det} 1,1105 \operatorname{det} 4,1472 \operatorname{det} 5,1228 \operatorname{det} 7$

b $22641 \operatorname{det} 1,10636 \operatorname{det} 4,6982 \operatorname{det} 5,16533 \operatorname{det} 7$

\section{SPEAKer Verification SETUP}

\section{A. Corpora and Classifiers}

For the speaker verification experiments, we utilize two different corpora and three classifiers (Table I). We use the NIST 2002 speaker recognition evaluation (SRE) corpus for extensive exploration of control parameters and effect of additive noise. For this, we employ a lightweight Gaussian mixture model with universal background model (GMMUBM) method [12] with test normalization (T-norm) [41]. The same system was used in our recent studies [33], [43]-[45]. Here we use it for choosing the type of multitaper variant for the more expensive NIST 2008 experiments. All the data in NIST 2002 contains telephone conversations collected over the cellular network.

We then verify our findings on an independent, more recent and much larger NIST 2008 SRE corpus which includes telephone, interview and auxiliary microphone data. For the experiments on NIST 2008 data, we employ two classifiers which were developed in participation of the past two NIST SRE campaigns [46]. The first system (GMM-SVM) uses Gaussian mean supervectors with support vector machine (SVM) [13] and nuisance attribute projection (NAP) technique [14], [34] for channel compensation. Zero normalization followed by T-norm (ZT-norm) [42] is used for score normalization. The second system (GMM-JFA) is a widely recognized high performance system, which uses joint factor analysis (JFA) technique [15], [16], [35] for integrated intersession and speaker variability modeling in the GMM supervector space. 
For score normalization, we use T-norm followed by Z-norm (TZ-norm).

For the recognition experiments under additive noise degradation, we contaminate the test utterances with factory noise while the background, cohort and target models are kept untouched. In [47] we found spectral subtraction [48] to be useful under additive noise degradation and it is thus included in the NIST 2002 experiments. We also did preliminary evaluation on the NIST 2008 data but the improvement was not systematic, and, given the added computational overhead, we decided not to include it to the NIST 2008 experiments.

\section{B. Performance Evaluation}

In comparison of the different MFCC estimators, we evaluate speaker verification accuracy using equal error rate (EER) and minimum detection cost function (MinDCF). EER is the error rate at the threshold $\theta_{\mathrm{EER}}$ for which the miss and false alarm rates are equal: $\mathrm{EER}=P_{\mathrm{miss}}\left(\theta_{\mathrm{EER}}\right)=P_{\mathrm{fa}}\left(\theta_{\mathrm{EER}}\right)$. MinDCF is used in the NIST speaker recognition evaluations and is defined as $\min _{\theta}\left\{C_{\text {miss }} P_{\text {miss }}(\theta) P_{\text {tar }}+C_{\mathrm{fa}} P_{\mathrm{fa}}(\theta)\left(1-P_{\mathrm{tar}}\right)\right\}$, where $C_{\text {miss }}=10$ is the cost of a miss (false rejection), $C_{\mathrm{fa}}=1$ is the cost of a false alarm (false acceptance) and $P_{\mathrm{tar}}=0.01$ is the prior probability of a target (true) speaker. In addition, we show selected detection error tradeoff (DET) plots [49] for the entire trade-off of false alarm and miss rates.

\section{Feature Extraction}

For the baseline Hamming method, we compute the MFCCs using the typical procedure [4]: Hamming window (frame duration $30 \mathrm{~ms}$ and hop $15 \mathrm{~ms}$ ), DFT spectrum estimate using windowed periodogram (Eq. 1), 27-channel mel-frequency filterbank, logarithmic compression and discrete cosine transform (DCT). We retain the lowest 18 MFCCs, excluding the energy coefficient $c_{0}$. For Thomson [24], multipeak [26] and sine-weighted cepstrum estimator (SWCE) [9] methods, the steps are the same, except that the spectrum is estimated using Eq. (2). In preliminary experiments, we found the frequently used pre-emphasis filter $H(z)=1-0.97 z^{-1}$ to degrade accuracy and it is therefore turned off in all the experiments.

After the 18 base MFCCs are extracted, we apply RASTA filter [5] and append the $\Delta$ and $\Delta^{2}$ coefficients, implying 54dimensional features. We then discard the nonspeech frames using an energy-based voice activity detector (VAD) and carry out utterance-level cepstral mean and variance normalization (CMVN). RASTA and CMVN are used for mitigating linear channel distortions.

We were also curious to see the effect of excluding the MFCC filterbank and to compute the 18 coefficients directly from the unwarped spectrum. We hypothesized that the double smoothing of multitaper spectrum followed by mel-filter energy integration might be suboptimal for speaker verification where we wish to retain the spectral details in addition to the envelope. We address this hypothesis on the NIST 2002 corpus in subsection VI-A.
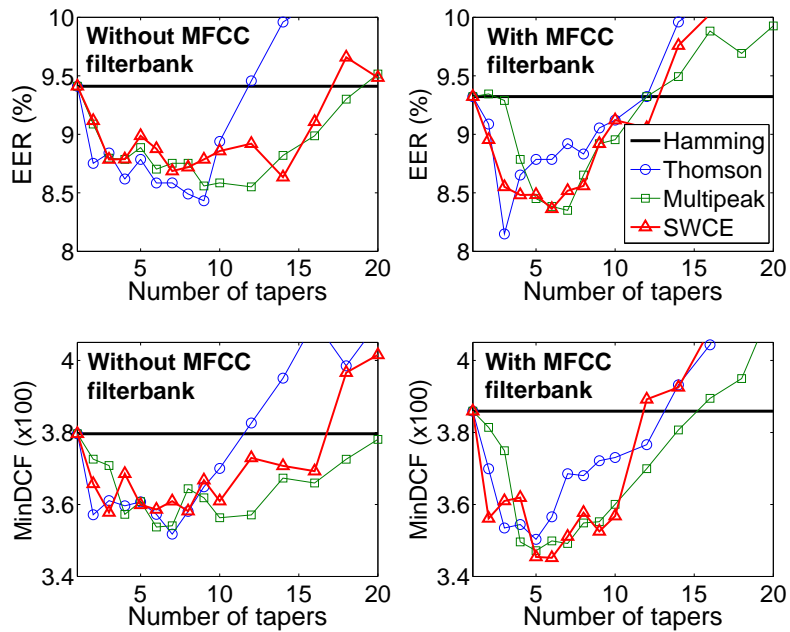

Fig. 6. Effects of the number of tapers and MFCC filterbank to different methods.

\section{Speaker Verification Results}

\section{A. GMM-UBM system on the NIST 2002 SRE corpus}

We first study how the choice of the spectrum estimation method affects speaker verification accuracy. For each of the multitaper methods - Thomson, multipeak and SWCE - we vary the number of tapers and contrast the result to the baseline Hamming method. EER and MinDCF, for both with and without MFCC filterbank, are shown in Fig. 6 where the horizontal (black) line represents the baseline. We observe the following:

- Multitaper methods outperform Hamming in both EER and MinDCF for a wide range of taper count (approx. $2 \leq K \leq 10$ ). Optimum value of $K$ depends on the method and the objective (EER or MinDCF).

- By including the MFCC filterbank the optimum points shift to left (less tapers) in most cases. This is expected because the MFCC filterbank introduces additional averaging over multitapering. Using MFCC filterbank improves EER and MinDCF and makes the curves generally less ragged, indicating stable parameter setting.

- The performance of the three multitaper methods at their optima are close to each other. Thomson shows sharper local mimima than multipeak and SWCE methods and gives higher error rates for large number of tapers.

The trends in Fig. 6 are, interestingly, in a reasonable agreement with Fig. 5. Both MSE, EER and MinDCF demonstrate approximately convex shapes and all the three methods give similar performance with optimized $K$. Secondly, for large $K$, MSE(Thomson) > MSE(SWCE) $\approx$ MSE(Multipeak); the same approximate ordering holds also for EER and MinDCF.

We next study the accuracy under additive factory noise corruption. Based on Fig. 6, for each method, we set the number of tapers to give both small EER and MinDCF. For the nonwarped case (no MFCC filterbank) we set the values to $K=8$ (Thomson), $K=10$ (multipeak) and $K=7$ (SWCE). For the warped frequency case (MFCC filterbank included), in turn, we set the values to $K=3$ (Thomson), $K=5$ 
TABLE II

RESULTS UNDER FACTORY NOISE CORRUPTION ON THE NIST 2002 CORPUS CORRESPONDING TO THE RIGHT HAND SIDE PLOTS (MFCC FILTERBANK INCLUDED) OF FIG. 7. IN EACH ROW, THE ERROR COUNTS SIGNIFICANTLY DIFFERING FROM THE BASELINE HAMMING, USING MCNEMAR'S TEST AT 95 $\%$ CONFIDENCE LEVEL, ARE INDICATED FOR BOTH GENUINE $(\bullet)$ AND IMPOSTOR $(\dagger)$ TRIALS.

\begin{tabular}{|c|c|c|c|c|c|c|c|c|c|c|c|c|c|}
\hline \multirow{2}{*}{\begin{tabular}{|l} 
SNR \\
$(\mathrm{dB})$
\end{tabular}} & \multicolumn{6}{|c|}{ Equal error rate (EER, \%) } & \multicolumn{7}{|c|}{ MinDCF $(\times 100)$} \\
\hline & Hamming & Thomson & & Multip. & & SWCE & Hamming & Thomson & & Multip. & & SWCE & \\
\hline Orig. & 9.32 & 8.15 & & 8.45 & - & $8.36 \bullet$ & \begin{tabular}{|l}
3.86 \\
\end{tabular} & 3.53 & $\bullet \dagger$ & 3.47 & $\bullet \dagger$ & 3.45 & \\
\hline 20 & & & - $\dagger$ & & - & 8.69 & & & - $\dagger$ & 3.62 & 1 & & - \\
\hline 10 & & & $\dagger$ & 9.66 & & 9.62 & & & - $\dagger$ & 4.11 & $\dagger$ & 3 & - \\
\hline 0 & & & & & & & & & - $\dagger$ & & - $\dagger$ & & \\
\hline-10 & 17.17 & 16.52 & & 15.86 & & 15.96 & 7.38 & 7.04 & & 6.72 & & 6.49 & \\
\hline
\end{tabular}
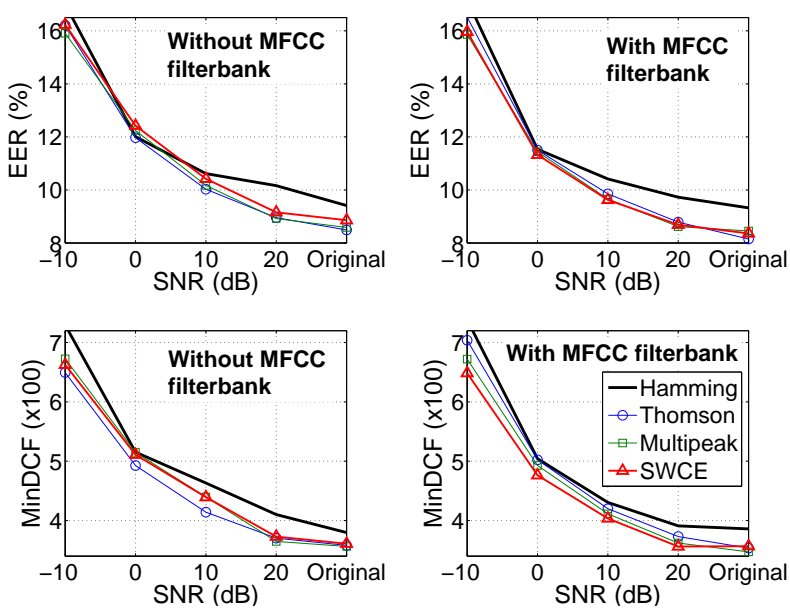

Fig. 7. Effect of factory noise under different signal-to-noise ratios.
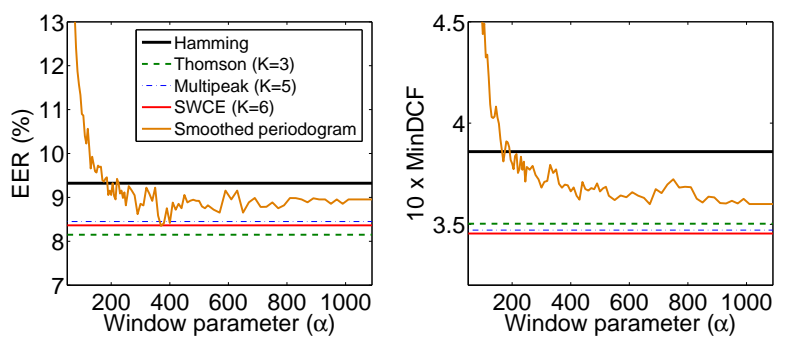

Fig. 8. Periodogram smoothing on the NIST 2002 corpus. As a reference, results for Hamming, Thomson, multipeak and SWCE method are also given. Smoothing is performed by convolving the unwindowed periodogram with a Gaussian kernel with parameter $\alpha$ controlling the kernel width.

(multipeak) and $K=6$ (SWCE). The results, as a function of SNR, are given in Fig. 7. The following can be observed:

- Accuracy of all methods drops as SNR decreases, as expected. Multitapers outperform Hamming in nearly all cases (the exception occurs at $0 \mathrm{~dB}$ but the EER difference is not statistically significant, see Table II)

- In the noisy cases (SNR $\leq 20 \mathrm{~dB}$ ), Thomson performs best on average when mel-warping is not applied; for the mel-warped case, SWCE performs the best.

- MFCC filterbank improves both EER and MinDCF.

Table II further displays the exact error values for the melwarped case. We also carry out McNemar's significance testing with $95 \%$ confidence level at both operating points [4], [50]. In 27 out of 30 cases, the difference between the multitaper and the baseline is significant in at least one of the error types.
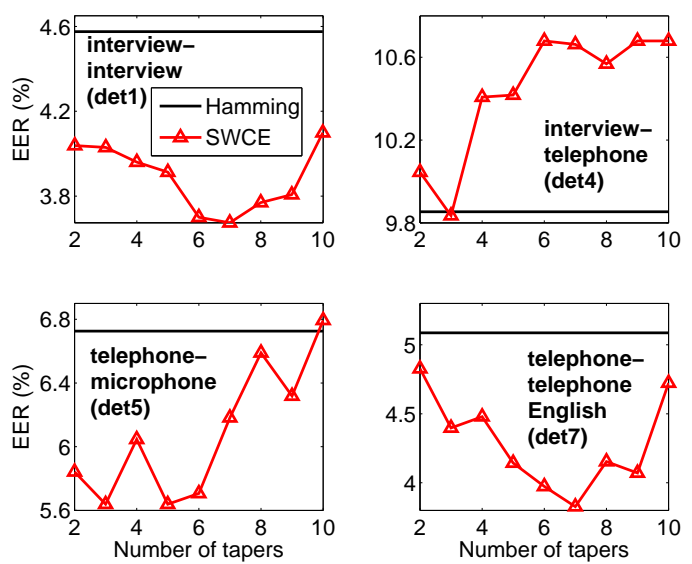

Fig. 9. Equal error rates (EER) on the NIST 2008 core task.
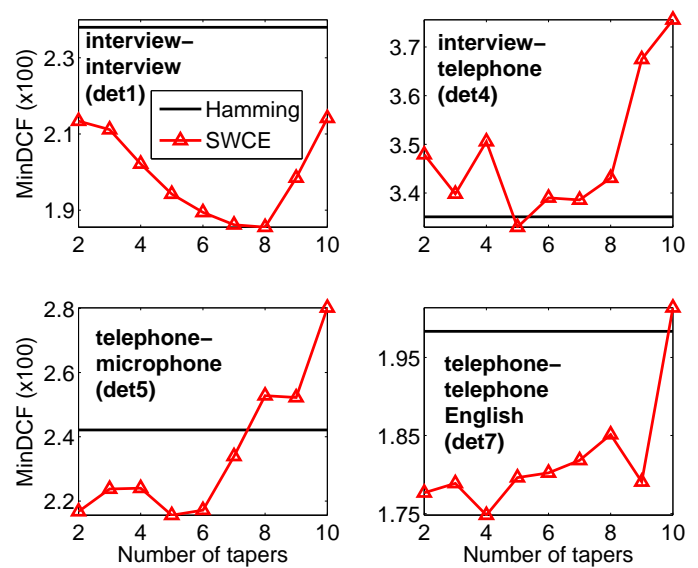

Fig. 10. MinDCF values on the NIST 2008 core task.

\section{B. Comparison with Periodogram Smoothing}

Due to its popularity in other application domains, we are interested in the performance of periodogram smoothing, i.e. convolution of $|X(f)|^{2}$ with a frequency-domain smoothing kernel. As discussed in [10], choice of the kernel (in particular, its bandwidth) is not easy but typically requires trial-anderror for a given application. To this end, we convolve the unwindowed periodogram estimate with a Gaussian window ${ }^{1}$

${ }^{1}$ Choice of window is less important than its bandwidth [10]. We use Matlab's gausswin $(N, \alpha)$ command, with $N=512$. 
$w(n)=\exp \left\{-\frac{1}{2}\left(\alpha \frac{n}{N / 2}\right)^{2}\right\}$, where $N$ and $\alpha$ are the size and width of the window, respectively. The width of the window is inversely related to the value of $\alpha$; a larger value of $\alpha$ produces a narrower window. The result is displayed in Fig. 8 for the same configuration as Fig. 6 for the mel-warped case. As a reference, we show the optimized results for Hamming, Thomson, multipeak and SWCE methods from Fig. 6.

By optimizing $\alpha$, periodogram smoothing outperforms the baseline Hamming method, but it does not outperform any of the multitaper methods. For $\alpha \approx 400$ (for which the effective number of non-zero samples in the kernel is about 4), EER is close to those of the SWCE and multipeak methods. But for the primary metric of speaker recognition evaluations, MinDCF, multitapers perform better.

\section{Experiments on the NIST 2008 SRE Corpus}

Due to expensive nature of NIST 2008 experiments, we fix as many parameters as we think reasonable. We choose to use SWCE method with MFCC filterbank based on observations from Fig. 7. We first verify our observations regarding suitable number of tapers, using the GMM-SVM system. The EER and MinDCF values in Figs. 9 and 10 indicate that, even though setting depends on the data condition, the optima are always achieved with $3 \leq K \leq 8$. This range agrees well with the NIST 2002 GMM-UBM result in Fig. 6, which has a completely different classifier, implementation details and choice of data sets. SWCE outperforms Hamming for a wide range of $K$ and therefore, the exact setting does not appear very critical.

In one of the sub-conditions ( $\operatorname{det} 4)$, the baseline Hamming outperforms multitaper. One reason might be non-optimal selection of datasets for channel compensation in this subcondition; the error rates for both Hamming and SWCE are higher than those in the other three conditions.

TABLE III

RESULTS ON THE DIFFERENT SUB-CONDITIONS OF THE NIST SRE 2008 CORE TASK (SHORT2-SHORT3) USING $K=6$ TAPERS. DET $1=$ INTERVIEW TRAINING AND TESTING; DET4 = INTERVIEW TRAINING, TELEPHONE TESTING; DET $5=$ TELEPHONE TRAINING, NON-INTERVIEW MICROPHONE TESTING; DET7 $=$ TELEPHONE TRAINING AND TEST INVOLVING ENGLISH LANGUAGE ONLY.

\begin{tabular}{|l|c|c|c|c|c|c|}
\hline & \multicolumn{3}{|c|}{ EER (\%) } & \multicolumn{3}{c|}{$100 \times$ MinDCF } \\
\cline { 2 - 6 } & Hamm. & SWCE & Impr. (\%) & Hamm. & SWCE & Impr. (\%) \\
\hline \hline \multicolumn{7}{|c|}{ GMM-SVM recognizer } \\
\hline det1 & 4.58 & 3.70 & 19.1 & 2.38 & 1.89 & 20.4 \\
det4 & 9.85 & 10.68 & -8.4 & 3.35 & 3.39 & -1.2 \\
det5 & 6.73 & 5.71 & 15.2 & 2.42 & 2.17 & 10.3 \\
det7 & 5.09 & 3.97 & 21.9 & 1.98 & 1.80 & 9.1 \\
\hline \hline \multicolumn{7}{|c|}{ GMM-JFA recognizer } \\
\hline det1 & 5.36 & 4.73 & 11.8 & 2.95 & 2.55 & 13.7 \\
det4 & 7.51 & 6.24 & 16.9 & 3.14 & 2.82 & 10.2 \\
det5 & 6.79 & 5.91 & 13.0 & 2.45 & 2.23 & 9.1 \\
det7 & 3.58 & 3.48 & 2.9 & 1.58 & 1.28 & 18.7 \\
\hline
\end{tabular}

In the following, we fix $K=6$ for all the four subconditions and for both genders. Gender-pooled results (without any score calibration) for each subcondition are given in Table III for both recognizers. Additionally, Fig. 11 displays the DET plot
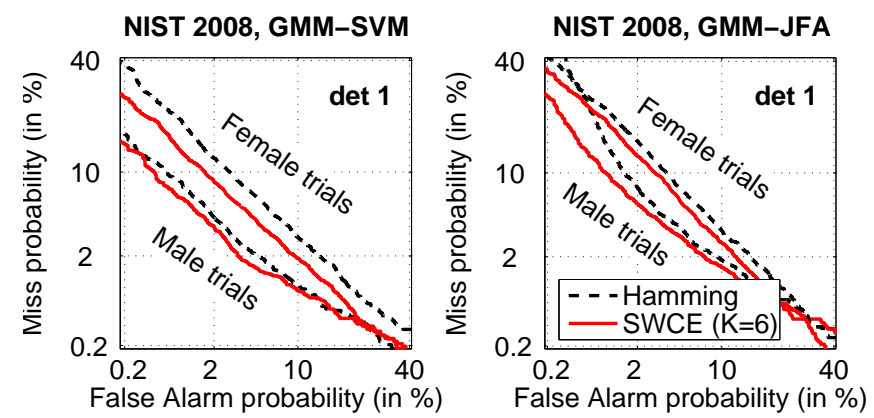

Fig. 11. DET plots for the interview-interview data (det1) on NIST 2008. The SWCE method uses $K=6$ tapers.

for the interview-interview condition (det1). From Table III, we observe the following.

- Except for det4 in GMM-SVM system, SWCE systematically outperforms Hamming in both EER and MinDCF.

- For GMM-SVM, det7 task was observed the highest EER improvement of $21.9 \%$, while det1 task the highest MinDCF improvement of $20.4 \%$. The largest overall improvements are in det 1 where both metrics decrease by about $20 \%$.

- For GMM-JFA, det4 task was observed the highest EER improvement of $16.9 \%$, while det7 task the highest MinDCF improvement of $18.7 \%$.

The DET plots in Fig. 11 further confirm that both recognizers benefit from multitapering over a wide range of operating points. Our JFA result is roughly on the same range as other similar systems, such as the full JFA system in [19, Table IX]. The $i$-vector system in [19] outperforms our JFA result on det7 (for instance, EERs of $2.9 \%$ and $1.1 \%$ were reported for male and female trials). Since i-vector and GMM-JFA share almost the same components - factor analysis on GMM supervectors with eigenvoice adaptation - we expect the results, to a certain extent, to generalize to i-vector classifier as well. In fact, preliminary indication of this was recently given in [51] on the SRE 2010 corpus using an independent implementation.

\section{CONCLUSIONS}

We have advocated the use of multitaper MFCC features in speech processing. By replacing the windowed DFT with multitaper spectrum estimate, we found systematic improvements in three independently constructed recognition systems (GMM-UBM, GMM-SVM and GMM-JFA). The improvements were consistent on two very different corpora (NIST 2002 and NIST 2008) including telephony, microphone and interview segments with severe cross-channel variabilities. These observations, together with analysis of bias and variance on TIMIT, gives us confidence to recommend using multitapers in speaker verification and possibly other speech processing tasks.

The choice of the multitaper type (Thomson, multipeak, SWCE) was found less important than the choice of the number of tapers, $K$. But even the exact choice of $K$ does 
[30] P.D. Welch. The use of Fast Fourier Transform for the estimation of power spectra: a method based on time averaging over short, modified periodograms. IEEE Transactions on Audio and Electroacoustics, AU15(2):70-73, June 1967

[31] C.H. Shadle and G. Ramsay. Multitaper analysis of fundamental frequency variations during voiced fricatives. In Proc. 6th Int. Seminar on Speech Production, pages CD-6, December 2003.

[32] N. Erdol and T. Gunes. Multitaper covariance estimation and spectral denoising. In Signals, Systems and Computers, 2005. Conference Record of the Thirty-Ninth Asilomar Conference on, pages 1144 - 1147, November 2005

[33] T. Kinnunen, R. Saeidi, J. Sandberg, and M. Hansson-Sandsten. What else is new than the Hamming window? robust MFCCs for speaker recognition via multitapering. In Proc. Interspeech 2010, pages 27342737, Makuhari, Japan, September 2010.

[34] A. Solomonoff, W.M. Campbell, and I. Boardman. Advances in channel compensation for SVM speaker recognition. In Proc. Int. Conf. on Acoustics, Speech, and Signal Processing (ICASSP 2005), pages 629632, Philadelphia, USA, March 2005.

[35] P. Kenny. Joint factor analysis of speaker and session variability: theory and algorithms. technical report CRIM-06/08-14, 2006.

[36] P. Kenny, G. Boulianne, P. Ouellet, and P. Dumouchel. Speaker and session variability in GMM-based speaker verification. IEEE Trans. Audio, Speech and Language Processing, 15(4):1448-1460, May 2007.

[37] David J. Thomson. Jackknifing multitaper spectrum estimates. IEEE Signal Processing Magazine, 24(4):20-30, 2007.

[38] T. Gerkmann and R. Martin. On the statistics of spectral amplitudes after variance reduction by temporal cepstrum smoothing and cepstral nulling. IEEE Trans. on Signal Proc., 57(11):4165-4174, Nov 2009.

[39] Gideon Schwarz. Estimating the dimension of a model. The Annals of Statistics, 6:461-464, March 1978.

[40] T. Schneider and A. Neumaier. Algorithm 808: ARfit - a Matlab package for the estimation of parameters and eigenmodes of multivariate autoregressive models. ACM Trans. Math. Softw., 27:58-65, 2001.

[41] R. Auckenthaler, M. Carey, and H. Lloyd-Thomas. Score normalization for text-independent speaker verification systems. Digital Signal Processing, 10(1-3):42-54, January 2000.

[42] R. Vogt, B. Baker, and S. Sridharan. Modelling session variability in text-independent speaker verification. In Proc. Interspeech 2005, pages 3117-3120, Lisboa, Portugal, September 2005.

[43] R. Saeidi, H.R.S. Mohammadi, T. Ganchev, and R.D.Rodman. Particle swarm optimization for sorted adapted Gaussian mixture models. IEEE Trans. Audio, Speech and Language Processing, 17(2):344-353, February 2009.

[44] R. Saeidi, J. Pohjalainen, T. Kinnunen, and P. Alku. Temporally weighted linear prediction features for tackling additive noise in speaker verification. IEEE Signal Processing Letters, 17(6):599-602, 2010.

[45] J. Pohjalainen, R. Saeidi, T. Kinnunen, and P. Alku. Extended weighted linear prediction (xlp) analysis of speech and its application to speaker verification in adverse conditions. In Proc. Interspeech 2010, pages 1477-1480, Makuhari, Japan, September 2010.

[46] H. Li, B. Ma, K.-A. Lee, H. Sun, D. Zhu, K.C. Sim, C. You, R. Tong, I. Kärkkäinen, C.-L. Huang, V. Pervouchine, W. Guo, Y. Li, L. Dai, M. Nosratighods, T. Tharmarajah, J. Epps, E. Ambikairajah, E.-S. Chng, T. Schultz, and Q. Jin. The I4U system in NIST 2008 speaker recognition evaluation. In Proc. Int. conference on acoustics, speech, and signal processing (ICASSP 2009), pages 4201-4204, Taipei, Taiwan, April 2009.

[47] R. Saeidi, J. Pohjalainen, T. Kinnunen, and P. Alku. Temporally weighted linear prediction features for speaker verification in additive noise. In Odyssey 2010: The Speaker and Language Recognition Workshop, Brno, Czech Republic, June 2010.

[48] P.C. Loizou. Speech Enhancement: Theory and Practice. CRC Press, 2007.

[49] A. Martin, G. Doddington, T. Kamm, M. Ordowski, and M. Przybocki. The DET curve in assessment of detection task performance. In Proc. 5th European Conference on Speech Communication and Technology (Eurospeech 1997), pages 1895-1898, Rhodos, Greece, September 1997.

[50] D.A. van Leeuwen, A.F. Martin, M.A. Przybocki, and J.S. Bouten. NIST and NFI-TNO evaluations of automatic speaker recognition. Computer Speech and Language, 20:128-158, April-July 2006.

[51] Md. J. Alam, T. Kinnunen, P. Kenny, P. Ouellet, and D. O'Shaughnessy. Multitaper MFCC features for speaker verification using i-vectors. In Proc. IEEE Automatic Speech Recognition and Understanding (ASRU 2011), pages 547-552, Hawaii, December 2011.

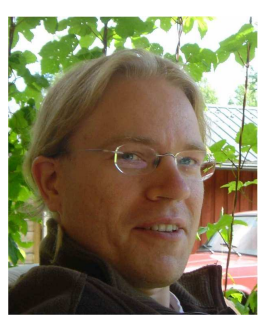

Tomi Kinnunen received the M.Sc. and Ph.D. degrees in computer science from the University of Joensuu, Finland, in 1999 and 2005, respectively. He worked as an associate scientist at the Institute for Infocomm Research $\left(\mathrm{I}^{2} \mathrm{R}\right)$, Singapore and as a senior assistant at the Department of Computer Science and Statistics, University of Joensuu, Finland. He is currently employed by the Academy of Finland as a post-doctoral researcher. His research areas cover speaker recognition and speech signal processing.

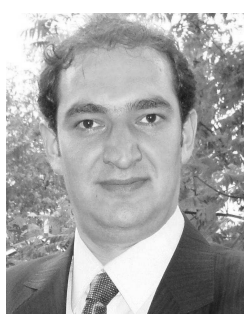

Rahim Saeidi (S'09) received B.Sc. degree in electrical engineering from Azad University-Save branch, Saveh, Iran, in 2002, and M.Sc. degree in telecommunication systems engineering from the Iran University of Science and Technology, Tehran, Iran, in 2005. He received his $\mathrm{PhD}$ degree from the University of Eastern Finland in 2011 and works currently as a postdoctoral researcher in Radboud University Nijmegen, Netherlands. His research interests include speech processing, machine learning, neuroscience and pattern recognition.

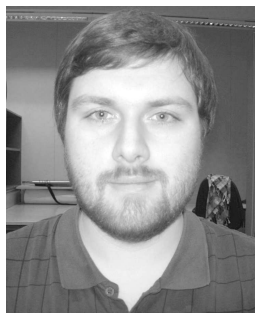

Filip Sedlák was born in 1985 in Brno, Czech Republic. He received his $\mathrm{BSc}$ degree from the Faculty of Information Technology (FIT) in Brno University of Technology (BUT). In 2008, he moved to University of Eastern Finland (UEF) as an exchange student. From February 2010 to July 2010 , he was visiting Institute for Infocomm Research (I2R) and Nanyang Technological University (NTU), Singapore, as an intern. He is currently pursuing his master's degree in the School of Computing at UEF.

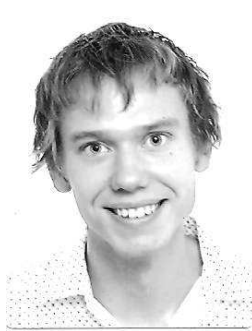

Johan Sandberg was born in 1980 in Sweden. He received the M.Sc. degree in Engineering Physics in 2005 and the Ph.D. degree in Mathematical Statistics in 2010, both at the Centre for Mathematical Sciences at Lund University, Sweden. His research field has included time-frequency analysis of time series, spectrum and cepstrum estimation and speech analysis. He is currently employed by Nordea Bank, Copenhagen, where his research interest is focused on modeling of currency exchange rates and interest rates.

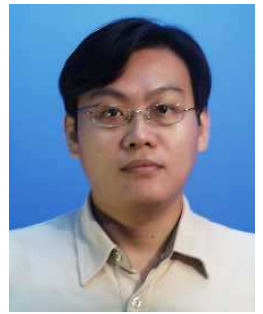

Kong Aik Lee received his B. Eng. (first class honors) degree from University Technology Malaysia in 1999, and his Ph.D. degree from Nanyang Technological University, Singapore, in 2006. He is currently a senior research fellow with Human Language Technology department, Institute for Infocomm Research $\left(\mathrm{I}^{2} \mathrm{R}\right)$, Singapore. His research focuses on statistical methods for speaker and spoken language recognition, adaptive echo and noise control, and subband adaptive filtering. He is the leading author of the book: Subband Adaptive Filtering. Theory and Implementation, Wiley, 2009.

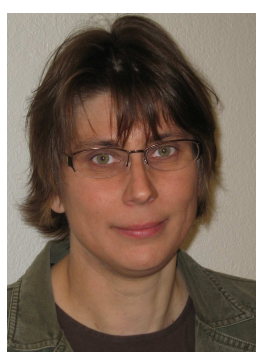

Maria Hansson-Sandsten (S'90-M'96) was born in Sweden 1966. She received the MSc degree in Electrical Engineering in 1989 and the $\mathrm{PhD}$ degree in Signal Processing in 1996, both from Lund University, Lund, Sweden. Currently she is Professor in Mathematical Statistics with speciality towards Statistical Signal Processing at the Centre for Mathematical Sciences, Lund University. Her current research interests include multitaper spectrum analysis and time-frequency analysis of stochastic processes with application areas of electroencephalogram signals, heart rate variability signals and speech signals. 


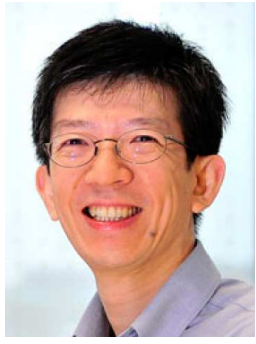

Dr Haizhou Li is currently the Principal Scientist and Department Head of Human Language Technology at the Institute for Infocomm Research. He is also the Program Manager of Social Robotics at the Science and Engineering Research Council of A*Star in Singapore.

Dr Li has worked on speech and language technology in academia and industry since 1988 . He taught in the University of Hong Kong (1988-1990),

South China University of Technology (1990-1994), and Nanyang Technological University (2006-). He was a Visiting Professor at CRIN/INRIA in France (1994-1995), and at the University of New South Wales in Australia (2008). As a technologist, he was appointed as Research Manager in Apple-ISS Research Centre (1996-1998), Research Director in Lernout \& Hauspie Asia Pacific (1999-2001), and Vice President in InfoTalk Corp. Ltd (2001-2003).

Dr Li's research interests include automatic speech recognition, natural language processing and information retrieval. He has published over 150 technical papers in international journals and conferences. He holds five international patents. Dr Li now serves as an Associate Editor of IEEE Transactions on Audio, Speech and Language Processing, and Springer International Journal of Social Robotics. He is an elected Board Member of the International Speech Communication Association (ISCA, 2009-2013), a Vice President of the Chinese and Oriental Language Information Processing Society (COLIPS, 2009-2011), an Executive Board Member of the Asian Federation of Natural Language Processing (AFNLP, 2006-2010), and a Senior Member of IEEE since 2001. Dr Li was the local arrangement chair of SIGIR 2008 and ACL-IJCNLP 2009. He was the recipient of National Infocomm Award of Singapore in 2001. He was named one of the two Nokia Professors 2009 by Nokia Foundation in recognition of his contribution to speaker and language recognition technologies. 